Z. REN, Z, SUN, Y, LI, X, FAN, M. DAI, Y, WANG, X. HU* (NORTHWEST UNIVERSITY XI'AN, P. R. OF CHINA)

Total Synthesis of (+)-3-Deoxyfortalpinoid F, (+)-Fortalpinoid A, and (+)-Cephinoid $\mathrm{H}$

Angew. Chem. Int. Ed. 2021, 60, 18572-18576, DOI: 10.1002/anie.202108034.

\section{Total Synthesis of (+)-Fortalpinoid A and (+)-Cephinoid H}

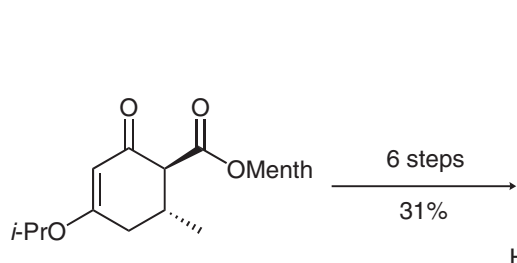

A

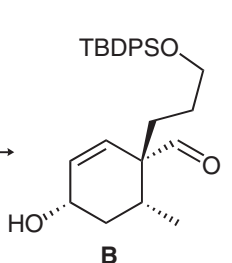

1. $\mathrm{Cu}(\mathrm{OTf})_{2}(10 \mathrm{~mol} \%), \mathrm{MgSO}_{4}$ i-PrOH$-\mathrm{CH}_{2} \mathrm{Cl}_{2}(1: 1), 50{ }^{\circ} \mathrm{C}$

2. TBAF, THF, $0^{\circ} \mathrm{C}$ to r.t.

3. TEMPO (10 mol\%), BAIB, $\mathrm{CH}_{2} \mathrm{Cl}_{2}$

4. C, THF, $-78{ }^{\circ} \mathrm{C}$

5. DMP, $\mathrm{NaHCO}_{3}, \mathrm{CH}_{2} \mathrm{Cl}_{2}$

$35 \%$
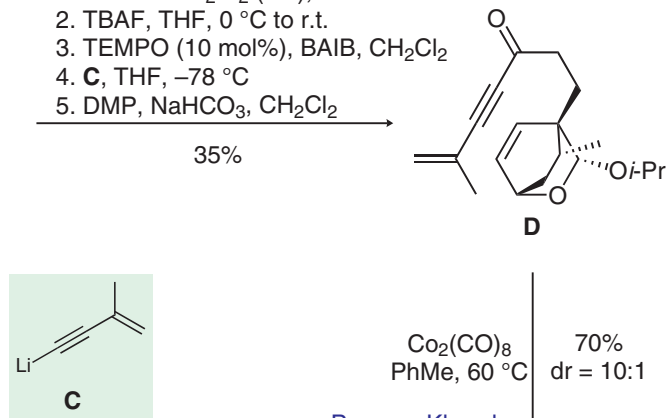

F, LDA, THF $-78^{\circ} \mathrm{C}$ then $\mathbf{E}$

2. TMSOTf, 2,6-lutidine, $\mathrm{CH}_{2} \mathrm{Cl}_{2}, 0^{\circ} \mathrm{C}$

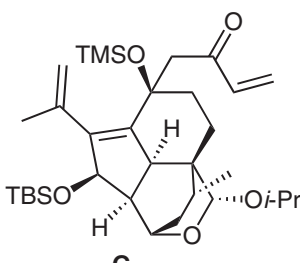

3. $\mathrm{LAH}, \mathrm{THF}, 0^{\circ} \mathrm{C}$

4. TBSOTf, 2,6-lutidine, $\mathrm{CH}_{2} \mathrm{Cl}_{2}, 0^{\circ} \mathrm{C}$

5. $\mathrm{CH}_{2} \mathrm{CHMgBr}$, THF, $0^{\circ} \mathrm{C}$

6. DMP, $\mathrm{NaHCO}_{3}, \mathrm{CH}_{2} \mathrm{Cl}_{2}$

$29 \%$

\section{Category}

Synthesis of Natural

Products and

Potential Drugs

\section{Key words}

cephalotaxus

(+)-fortalpinoid A

(+)-cephinoid $\mathrm{H}$

Pauson-Khand

cyclization

ring-closing metathesis

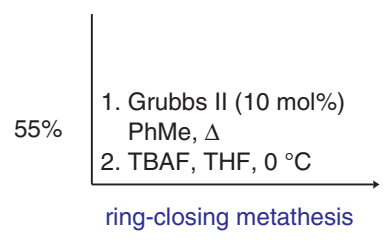

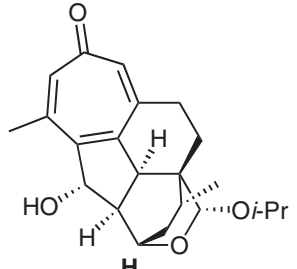

$\mathrm{H}$ $0^{\circ} \mathrm{C}$ to r.t.

2. $\mathrm{Bu}_{3} \mathrm{SnH}, \mathrm{AIBN}(10 \mathrm{~mol} \%)$ $\mathrm{PhMe}, \Delta$

3. $\mathrm{TsOH}$, acetone- $\mathrm{H}_{2} \mathrm{O}(4: 1)$

$50{ }^{\circ} \mathrm{C}$

4. $\mathrm{Ag}_{2} \mathrm{CO}_{3}$, Celite, PhMe, $\Delta$
Significance: Hu and co-workers report the first total synthesis of $(+)$-fortalpinoid $A$ and $(+)$-cephanoid $A$, members of a class of 17,17-norditerpenoids, isolated from Cephalotaxus fortunii.
Comment: Allyl alcohol B is accessed from vinylogous ester $\mathbf{A}$, first described by Myers and co-workers, and elaborated into enyne $\mathbf{D}$, which then undergoes the key carbonylative cyclization to furnish E and give access to three natural products.

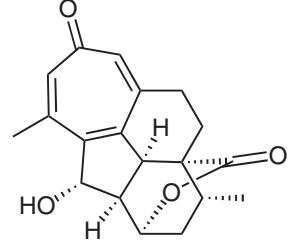

(+)-Fortalpinoid A 